\title{
Ulrike Zeuch \\ Das Gesunde und Kranke in der Literatur Robert Musil und die Krise der Moderne
}

Kunst kann Unanständiges und Krankes wohl zum Ausgangspunkt wählen, aber das daraufhin Dargestellte - nicht die Darstellung, sondern das dargestellte Unanständige und Kranke - ist weder unanständig mehr noch krank.

Musil stellt in seinem Essay Das Unanständige und Kranke in der Kunst (um Folgenden zitiert als Essay), der erstmals am 1. März 1911 im Pan erschien, ${ }^{1}$ die These auf, dass Kunst zwar «Unanständiges und Krankes» zum «Ausgangspunkt» wählen könne, das «daraufhin Dargestellte - nicht die Darstellung, sondern das dargestellte Unanständige und Kranke» - jedoch «weder unanständig mehr noch krank» sei. ${ }^{2}$ Musil bezeichnet seine These nicht als Annahme, die es noch zu beweisen gelten würde, sondern als Axiom. ${ }^{3}$ Dass er hier den Begriff Axiom verwendet, ist mehr als blosse Rhetorik oder strategisches Raffinement: Es ist Programm. Er verwendet einen Begriff, der durch David Hilbert, den Begründer der formalistischen, für das 20. Jahrhundert wegweisenden Logik 1889 eine radikal neue Bedeutung erhalten hat. ${ }^{4}$ In der sogenannten klassischen Axiomatik,

1 R. Musil: Das Unanständige und Kranke in der Kunst, in: Klagenfurter Ausgabe (im Folgenden zitiert als KA). Kommentierte Edition sämtlicher Werke, Briefe und nachgelassener Schriften, hg. von Walter Fanta u.a., Klagenfurt 2016ff., IX 135-144.

2 Ebd., 139.

3 Ebd.

4 David Hilbert: Grundlagen der Geometrie, Leipzig 1899; zu den ideengeschichtlichen und systematischen Hintergründen dieser Umdeutung vgl. 
von Euklids Geometrie ausgehend, gilt das Axiom als Ausgangsoder Grundsatz, der früher als das aus ihm Bewiesene ist und dieses begründet, sich selbst aber weder begründen noch deduktiv ableiten lässt. ${ }^{5}$ Laut Hilbert jedoch ist jede unabgeleitete Aussage als Aussageform ein Axiom; diese ist - bezogen auf eine Theorie, etwa ein axiomatisches System - an sich weder wahr noch falsch, ist (zunächst) unbewiesen, muss aber nicht zwingend unbewiesen sein bzw. bleiben. ${ }^{6}$ Als radikal neu, weder wahr noch falsch und nicht zwingend unbeweisbar - so will Musil seinen Essay verstanden wissen.

Im Folgenden werden die einzelnen Schritte von Musils Argumentation dargelegt und die Konsequenzen für die Kunst der Moderne skizziert, die sich aus seinem Axiom ergeben. Die Form des Essays gilt Musil als philosophisches Medium der Selbstreflexion, ${ }^{7}$ als Medium eines «lebendigen Denkens», ${ }^{8}$ das essayistische Schreiben als ein Schreiben ohne Denkverbot oder äussere und innere

Markus Schmitz: Analysis - eine Heuristik wissenschaftlicher Erkenntnis: platonisch-aristotelische Methodologie vor dem Hintergrund ihres rhetorisch-technisch beeinflussten Wandels in Mathematik und Philosophie der Neuzeit und Moderne, hg. von Wolfgang Bernard u.a., Freiburg im Breisgau 2010.

Axiom, in: Joachim Ritter (Hg.): Historisches Wörterbuch der Philosophie, Basel, Stuttgart 1971, I 737-748; dazu, dass der Begriff «Axiom» seit Aristoteles vielfältig umgedeutet worden ist, vgl. Nikolai N. Stuloff: Axiom, Exaktheit und Methodenreinheit. Historische Beiträge zum Wandel von Konzepten der Mathematik, mit einer Einleitung Erinnerungen an Nikolai Nikolaijewitsch Stuloff versehen und hg. von Fritz Krafft und mit einem Geleitwort von Christoph J. Scriba, Augsburg 2008.

Axiom, in: Jürgen Mittelstraß (Hg.): Enzyklopädie Philosophie und Wissenschaftstheorie, Stuttgart 22005, I 332-333; Ruth Bendels (Erzählen zwischen Hilbert und Einstein. Naturwissenschaft und Literatur in Hermann Brochs «Eine methodologische Novelle» und Robert Musils «Drei Frauen», Würzburg 2008) hebt hervor, dass die Gemeinsamkeit zwischen Literatur und Mathematik für Musil darin bestehe, «Systeme für spätere, noch unbekannte Ziele zu erfinden» (239).

7 Vgl. Birgit Nübel: Robert Musil. Essayismus als Selbstreflexion der Moderne, Berlin 2006.

8 Olav Krämer: Denken erzählen. Repräsentationen des Intellekts bei Robert Musil und Paul Valéry, Berlin 2009, 165-169. 
Zensur. ${ }^{9}$ Zunächst jedoch gilt es zu klären, was Musil unter unanständig und krank versteht, und diese Begriffe in den Kontext seines Werks und seiner Zeit zu setzen.

«Krank» und «unanständig» - Musils Begriffsbestimmung

Unter «krank» und «unanständig» versteht Musil die gesellschaftlich, das heisst moralisch geächtete und zugleich von der Gesetzgebung und der praktizierten Rechtsprechung aus sanktionierte Devianz im Sinne einer Abweichung von der Norm. ${ }^{10}$ Dabei hat er vor allem die sexuelle Devianz im Blick. ${ }^{11}$ Die Beispiele, die er dafür in seinem Essay anführt, sind Abweichungen, die sich im Bereich der Fantasie abspielen; sie werden nicht manifest, anders als zum Beispiel in den Verwirrungen des Zöglings Törless im physischen und psychischen sexuellen Missbrauch Basinis ${ }^{12}$ oder im Mann ohne Eigenschaften (im Folgenden zitiert als $M o E$ ) in den Sexualmorden Moosbruggers. ${ }^{13}$ Diese Devianz geschieht assoziativ, ereignet sich

9 Zsuzsa Bognár: «Als Mischprodukt verrufen». Der literarische Essay der Moderne, Wien 2017, zu Musils Kulturkritik im Essay: 133-168. Vgl. Birgit Nübel: Möglichkeitssinn und Essayismus, in: Birgit Nübel, Norbert Christian Wolf (Hg.): Robert Musil Handbuch, Berlin, Boston 2016, 719 725, sowie Andrea Pelmter: «Experimentierfeld des Seinkönnens» - Dichtung als «Versuchsstätte». Zur Rolle des Experiments bei Robert Musil, Würzburg 2008.

10 Vgl. zum zeitgenössischen Begriff der «Norm» bzw. «Normalität» Michaela Ralser: Das Subjekt der Normalität. Das Wissensarchiv der Psychiatrie. Kulturen der Krankheit um 1900, München 2010.

11 Zum Kontext der Zensur, Perversion, Pornographie, Psychoanalyse und Sexualwissenschaft in Musils Essay vgl. Birgit Nübel: «ein dünner Dunst fremden Leibes». Perversionen des Erkennens in Musils Essay «Das Unanständige und Kranke in der Kunst», in: Musil-Forum 31 / 10 (2009) 23-38.

12 R. Musil: Die Verwirrungen des Zöglings Törless, KA VII 11-220; vgl. Dorothee Kimmich: Die Verwirrungen des Zöglings Törless (1906), in: B. Nübel, N. Ch. Wolf: Robert Musil Handbuch, 101-111, hier 105.

13 Vgl. Mark Ludwig: Zurechnungsfähigkeiten. Kriminologie in Robert Musils Mann ohne Eigenschaften, Würzburg 2011, hier bes., 92-132 (Fallgeschichten) u. 133-146 (Kriminalphysiognomien). 
im Kopf, und zwar dann, wenn das Bewusstsein schwankt, ins Flattern kommt, erregt wird und sich «Halbvorgänge[n]» hingibt, die «nie vollendet, dennoch geschlechtlich, dennoch unerlaubt hier, dennoch prometheisch» zum ersten Mal dann fühlbar werden, wenn das Ich seine rationale Kontrolle für einen Moment preisgibt. ${ }^{14}$

Dieses Kino im Kopf, assoziativ, im Moment, wie von selbst, unwillkürlich, das heisst der Kontrolle des bewussten Ichs entzogen, passiert gerade in - von aussen betrachtet - scheinbar äusserst rationalen, linearen Vollzügen, etwa denen eines Chirurgen bei einem hoch konzentrierten operativen Eingriff:

[...] irgendeiner spricht - sachlich, wenig, medizinisch - ein Dirigierender, ein Herr und etwas liegt reglos dargeboten, eine Wunde, halb fremd hier, blumenhaft, halb blutig Schleimendes, geöffnet, mitten in der weissgespannten Haut der Seite, wie ein Mund ... Eine automatische Assoziation ... küssen, die wehrlose Haut der Lippen daraufpressen. Warum? Wer weiss es? Eine äussere Ähnlichkeit, eine Wehmut? ... Ein Sekundenteil Grauen darüber und dann wieder Kommandoworte und schnelle Handgriffe. ${ }^{15}$

Analog inszeniert Gottfried Benn mit seinem 1912 veröffentlichten Gedichtzyklus Morgue den Einbruch assoziativer, sexuell konnotierter Imaginationen in äusserlich rationale Handlungsvollzüge. ${ }^{16}$

R. Musil: Das Unanständige, KA IX 136.

15 Ebd. Zeitgleich erprobt Gottfried Benn (Prosa und Autobiografie. In der Fassung der Erstdrucke, hg. von Bruno Hillebrand, Frankfurt am Main 1984) in den Rönne-Novellen mit dem jungen Arzt Rönne - Pathologe und Chirurg in einer Lungenheilanstalt in Vertretung - den Austritt aus dem Bereich der Normalität; vgl. Gehirne (1914) und den Übertritt in den «Bereich der Möglichkeiten» (34), «in das Fragwürdige» (36), das «Ungeformte», das «Uferlose» (37), fern «zielstrebigen, kausal geleiteten Handelns» (38). In Der Geburtstag (1916) lässt Benn Rönne sinnieren: «Welches war der Weg der Menschheit gewesen bis hierher? Sie hatte Ordnung herstellen wollen in etwas, das hätte Spiel bleiben sollen. Aber schliesslich war es doch Spiel geblieben, denn nichts war wirklich. War er wirklich? Nein; nur alles möglich, das war er» (47). Vgl. Nicola Gess: Primitives Denken. Wilde Kinder und Wahnsinnige in der literarischen Moderne (Müller, Musil, Benn, Benjamin), München 2013.

16 Vgl. Alexandra Rassidakis: Gottfried Benn, «Morgue». Poetik der Präsenz, in: Peter Hanenberg u.a. (Hg.): Rahmenwechsel Kulturwissenschaften, Würzburg 2010, 207-216. 
Dass Musil ausgerechnet die sexuelle Devianz im Blick hat, hängt mit der zeitgenössischen Psychoanalyse Sigmund Freuds zusammen. Nicht explizit, aber implizit auf Freud bezogen, schreibt Musil in seinem Essay Psychologie und Literatur: Es gebe

psychologische Arbeiten, die wie Dichtungen sind. Es sind Beschreibungen pathologischer Seelenabläufe, die von einer wunderbaren Eindringlichkeit u. so stark gleichnishaft (für den «normalen» Leser) sind, dass der Zusatz von Deutung, der grosse Dichtungen aus ihnen machen würde, kaum entbehrt wird. ${ }^{17}$

Während die Gesellschaft des wilhelminischen oder habsburgischen Kaiserreichs diese Art der Abweichung als krank, als moralisch verwerflich, als unanständig verurteilt, ${ }^{18}$ sieht Musil in ihr gerade etwas Prometheisches. Prometheus, eine in der Rezeption seit der Antike ebenso schillernde wie ambivalent beurteilte Gestalt der griechischen Mythologie, steht hier wie bei Goethes Hymne Prometheus als Prototyp für den Tabubruch. Gebrochen wird jedoch nicht (mehr) ein Tabu der Götter, sondern das der Gesellschaft, die gottgleich moralisches Fehlverhalten meint sanktionieren zu können, aber nicht weniger als die griechischen Götter ihre Sanktionen auf Prinzipien gründet, die menschengemacht, mithin konstruiert und insofern hinterfragbar sind.

Weshalb Musil ausgerechnet die sexuelle Devianz im Blick hat, hat eine weit vor der zeitgenössischen Psychoanalyse Sigmund Freuds liegende grundlegende Ursache: die spätestens seit der sogenannten dunklen Seite der Aufklärung mit Johann Gottfried Herder, Heinrich von Kleist und den Romantikern einsetzende, mit Georg Büchner ${ }^{19}$ radikalisierte massive Kritik an der Rationalität, die als Reduktion des Menschen auf das cogito, auf das, dessen sich

17 R. Musil: Psychologie und Literatur, in: Gesammelte Werke, hg. von Adolf Frisé, Reinbek bei Hamburg 1978, II 1345-1347, hier 1347.

$18 \mathrm{Zu}$ den zeitgenössischen Diskursen um Kriminelle und sexuelle Abweichungen vgl. Yvonne Wübben: Verrückte Sprache. Psychiater und Dichter in der Anstalt des 19. Jahrhunderts, Paderborn 2012.

19 Vgl. Yvonne Wübben: Büchners «Lenz»: Geschichte eines Falls, Paderborn 2016. 
der Mensch bewusst ist, verstanden wird. ${ }^{20}$ Bereits in den Verwirrungen des Zöglings Törless heisst es:

Ein Gedanke, - er mag schon lange vorher durch unser Hirn gezogen sein, wird erst in dem Momente lebendig, da etwas, das nicht mehr Denken, nicht mehr logisch ist, zu ihm hinzutritt, so dass wir seine Wahrheit fühlen, jenseits von allen Rechtfertigungen, wie einen Anker, der von ihm aus ins durchblutete, lebendige Fleisch riss ... Eine grosse Erkenntnis vollzieht sich nur zur Hälfte im Lichtkreis des Gehirns, zur andern Hälfte in dem dunklen Boden des Innersten, und sie ist vor allem ein Seelenzustand, auf dessen äusserster Spitze der Gedanke nur wie eine Blüte sitzt. ${ }^{21}$

Schon in diesem Werk formuliert Musil den Gedanken, dass die Essenz des Menschen nicht in dem bestehe, was er denke, sondern in dem, was er fühle. Es handelt sich um eben jene Sphäre des Gefühls, der er im MoE, ohne bis zu seinem Tod am 15. April 1942 $\mathrm{zu}$ einer für ihn befriedigenden Antwort zu kommen, ${ }^{22}$ immer

Vgl. hierzu Ulrike Zeuch: Umkehr der Sinneshierarchie. Herder und die Aufwertung des Tastsinns seit der frühen Neuzeit, Tübingen 2000, 142-162. R. Musil: Törless, KA VII 215; vgl. Bernhard Greiner: Crimen - Diskriminierung - Literatur der Übertretung. Musil: «Die Verwirrungen des Zöglings Törless», Muschg: «Der Zusenn oder das Heimat», Kluge: «Warten auf bessere Zeiten», in: Joachim Linder u.a. (Hg.): Verbrechen Justiz - Medien. Konstellationen in Deutschland von 1900 bis zur Gegenwart, Tübingen 1999, 307-324.

22 R. Musil: Der Mann ohne Eigenschaften (im Folgenden zitiert als MoE), KA I-V; vgl. in IV die verschiedenen Versionen des Kapitels «Atemzüge eines Sommertages» (282-297 u. 426-438), die Kapitel «Agathe stösst zu ihrem Missvergnügen auf einen geschichtlichen Abriss der Gefühlspsychologie» (163-178), «Naive Beschreibung, wie sich ein Gefühl bildet» (193205), «Fühlen und Verhalten. Die Unsicherheit des Gefühls» (205-225), «Die Wirklichkeit und die Ekstase» (249-259) und «Ulrich und die zwei Welten des Gefühls» (260-271). Walter Fanta schreibt im Nachwort zum Band IV (441-463) zur Frage, warum der MoE unvollendet geblieben sei: «Wie die Erzählung der Geschwisterliebe ausginge, welche Entscheidung Ulrich angesichts des Kriegsausbruchs träfe, wollte Musil nicht a priori festgelegt wissen, sondern aus dem Ausgang der Gedankenexperimente bestimmen, die Ulrich in seinem sogenannten Tagebuch und die Geschwister in ihren Gesprächen anstellen» (446). Zu Ulrichs Denkweise im MoE und ihrer Veränderung vgl. O. Krämer: Denken erzählen, 230-291: «Für 
wieder umgeschriebene Kapitel gewidmet hat. Diese Sphäre des Gefühls beschreibt Musil auch als ein Umkehren des Fühlens, es gelte, die «Welt anders zu erleben», ${ }^{23}$ oder, wie in der Erzählung Die Amsel, als «einen zauberhaften Zustand ${ }^{24}$ «wie im Traum». ${ }^{25}$

Der Fall des Sexualmörders Moosbrugger. Oder: Wer ist hier krank?

Bevor Musil seinen Essay schrieb, hatte er sich eingehend mit dem Fall des Sexualstraftäters Christian Voigt befasst. ${ }^{26}$ Dieser hatte in Wien in der Nacht vom 13. auf den 14. August 1910 in einem

nicht-ratioide Gedanken ist Musil zufolge kennzeichnend, dass sie mit Gefühlen verbunden sind oder sich mit ihnen verbinden können. Auch die im Roman dargestellten Denkprozesse sind grossenteils mit Gefühlen verwoben. Das Verhältnis zwischen Gedanken und Gefühlen, wie es dort präsentiert wird, kann man in etwas zugespitzter und vereinfachender Weise so zusammenfassen: Gefühle bilden zum einen häufig den Anstoss $\mathrm{zu}$ Gedankengängen, insbesondere zu Gedankengängen, die auf eine Deutung oder Erklärung dieser Gefühle zielen; und Gefühle übernehmen zum anderen die Funktion der Bewertung von Gedanken.» (291) R. Musil: MoE, KA IV 268.

24 R. Musil: Die Amsel, KA VIII, Nachlass zu Lebzeiten (1935), 522-545, hier 528.

Ebd., 535. Vgl. hierzu Marie-Louise Roth: Wirklichkeit und Traum in Robert Musils «Nachlass zu Lebzeiten», in: Herbert Arlt, Manfred Diersch (Hg.): Sein und Schein - Traum und Wirklichkeit. Zur Poetik österreichischer Schriftsteller/innen im 20. Jahrhundert, Frankfurt am Main 1994, 63-84.

Karl Corino: Robert Musil. Eine Biographie, Reinbek bei Hamburg 2003, 880-891. Zu Musils lebenslangem Interesse an der Psychiatrie und zur Zeit der Abfassung des Essays an «sensationsträchtigen Strafrechtsprozessen» vgl. Yvonne Wübben: Psychiatrie, in: B. Nübel, N. Ch. Wolf: Robert Musil Handbuch, 524-530, hier 525. Als literarische Vorlage für Musil zentral ist Büchners Woyzeck, ein Dramenfragment, für dessen Abfassung Büchner eingehend den historischen Fall «Woyzeck» studiert und auf umfangreiches Material aus medizinischen und juristischen Gutachten zurückgegriffen hat; vgl. Dietrich von Engelhardt u.a. (Hg.): Der «Mord». Darstellung und Deutung in den Wissenschaften und Künsten, Lübeck 2007, 175-186 (zu Woyzeck) und 237-266 (zu Moosbrugger). 
Blutrausch eine Prostituierte erstochen, verstümmelt, mit dem Messer zerfleischt. Vor dieser Tat war Voigt bereits in Deutschland als Mörder bekannt gewesen und mehrere Jahre in der Irrenanstalt Bayreuth verwahrt worden. Wegen guter Führung war er 1909 als geheilt entlassen worden.

Was den Fall besonders prekär und für Musil interessant machte, war der Umstand, dass Voigt eine für die psychologische Frage der Zurechnungsfähigkeit und für die juristische Frage der Schuldfähigkeit komplexe Widersprüchlichkeit aufwies: einerseits durchaus intelligent, andererseits zu Jähzorn und unkontrollierten Ausbrüchen neigend. ${ }^{27}$ Die Richter verhängten auf der Grundlage eines medizinischen Gutachtens, das Voigt die vollumfängliche Schuldfähigkeit attestierte, über ihn die Todesstrafe; diese milderte Kaiser Franz Joseph am 22. Februar 1912 zu lebenslanger Haft ab. Das gesamte Verfahren, das Musil, seit Anfang 1911 in Wien, in der Presse verfolgt hatte, zeigte deutlich die Ambivalenz in der Bewertung von gesund und krank. ${ }^{28}$

Im $M o E$ stellte Musil diese Ambivalenz am Beispiel des Sexualmörders Moosbrugger, dessen Darstellung durch den Fall Christian Voigts inspiriert ist, im Kapitel «Ausflug ins logisch-sittliche Reich» folgendermassen dar: Alle im Gerichtssaal, Gerichtspsychiater, Juristen, Mediziner, Journalisten, wissen, dass «Moosbrugger in irgendeiner Weise krank» ist. ${ }^{29}$ Und dennoch folgern sie logisch:

https: / / www.nzz.ch/feuilleton/ robert-musil-und-der-frauenmoerderin-mann-ohne-eigenschaften-ld.1483798

Zum Themenkomplex Sexualität und Krankheit in Musils MoE vgl. Florian Kappeler: Situiertes Geschlecht. Organisation, Psychiatrie und Anthropologie in Robert Musils «Der Mann ohne Eigenschaften», München 2012; Martin Lindner: Der Mythos «Lustmord». Serienmörder in der deutschen Literatur, dem Film und der bildenden Kunst zwischen 1892 und 1932, in: Joachim Linder u.a. (Hg.): Verbrechen - Justiz Medien. Konstellationen in Deutschland von 1900 bis zur Gegenwart, Tübingen 1999, 273-306; zur Aktualität vgl. Ulrike Zeuch: Die Aktualität des Falls «Moosbrugger», in: Hans-Edwin Friedrich und Claus-Michael Ort (Hg.): Recht und Moral. Zur gesellschaftlichen Selbstverständigung über «Verbrechen» vom 17. bis zum 21. Jahrhundert, Berlin 2015, 399-419.

R. Musil: MoE, KA I 388. 
[...] wenn man teilweise krank ist, ist man nach Ansicht der Rechtslehrer auch teilweise gesund; ist man aber teilweise gesund, so ist man wenigstens teilweise zurechnungsfähig; und ist man teilweise zurechnungsfähig, so ist man es ganz; denn Zurechnungsfähigkeit ist [...] der Zustand des Menschen, in dem er die Kraft besitzt, unabhängig von jeder ihn zwingenden Notwendigkeit sich aus sich selbst für einen bestimmten Zweck zu bestimmen, und eine solche Bestimmtheit kann man nicht gleichzeitig besitzen und entbehren. ${ }^{30}$

Diese scheinbar so zwingende Logik hat jedoch eine Lücke, und auf diese Lücke will Musil hinaus: Nicht nur Moosbrugger, sondern jeder Mensch ist nicht nur rational, bewusst, kontrolliert, frei in seinen Handlungen, sondern jeder Mensch ist als derselbe zugleich auch irrational, unbewusst, unkontrolliert, unfrei in seinen Handlungen. Für Musil ist die Einschätzung des Unterschieds zwischen einem psychisch Kranken, der zum Straftäter oder gar zum Mörder wird, und dem seelisch Gesunden nur eine Frage der Perspektive:

Man hat über Determinirtheit $u$. Undeterminirtheit des menschlichen Handelns gestritten. Die Determinirtheit ist wissenschaftlich unabweisbar. Aber unter den Determinanten finden sich auch solche, die das Ich, die Persönlichkeit bilden. Es ist unhaltbar u. unnötig, dass das Ich die «Ursache» der Handlung sei, aber Ich $\mathrm{u}$. Handlung sind miteinander verknüpft u. das genügt. Der Grad dieser Verknüpfung sozusagen ist der Grad der Zurechenbarkeit. Weiters ist nun diese Verknüpfung beim Geisteskranken eine mehr innere, in den Fällen der Erfolghaftung eine mehr äussere. ${ }^{31}$

Welcher Teil also dieses Selbst wird da beurteilt und im Zweifelsfall verurteilt, und zwar verurteilt auf der Grundlage nicht minder ambivalenter Massstäbe? Und wer bestimmt, was sittlich, was anständig ist? Musil lässt Ulrich im $M o E$ auf diese Fragen folgendermassen antworten: Sitte und Anstand sind per Konsens etablierte moralische Massstäbe einer Gesellschaft; die Verletzung von Sitte und Anstand wird strafrechtlich verfolgt; wer diese verletzt, ist, so die Annahme, ein als verursachende Person in ihrer

$30 \quad$ Ebd., 388-389.

31 R. Musil: Quantificierbarkeit der Moral. Die Moral des Dichters (1910/11), in: Gesammelte Werke, hg. von Adolf Frisé, II 1307-1311, hier 1308. 
Selbstverantwortlichkeit konturiertes «Erfahrungssubjekt», ${ }^{32}$ weder aber sind diese Massstäbe wahr in Sinne von Allgemeingültigkeit noch gibt es ein so geartetes Subjekt; dieses ist vielmehr dezentriert. Folglich wird die Tat bestraft, den Täter kann der Richter, so Musil, als einzelne Person nicht kennen: «Man verlangt vom Richter, dass er ein Menschenkenner sei, aber es ist ihm ganz unmöglich, dass er das Individuum in seine Rechnung einstellt, es ist ihm nur das Speciesmässige zugänglich.» ${ }^{33}$

Indem Musil behauptet, der Unterschied zwischen einem psychisch Kranken, in diesem Fall sogar einem Sexualmörder, und einem psychisch Gesunden bestehe lediglich in der Unterschiedlichkeit des Ortes, an welchem Ich und Handlung miteinander verknüpft würden, im Inneren oder im Äusseren der menschlichen Seele, dringt er in «Bezirke [vor], die man nie [zuvor] betreten hat», wie es im Essay heisst. ${ }^{34}$ Dass die Grenzen zwischen bewusst und unbewusst, frei und unfrei, rational und irrational fliessend seien - davon ist die zeitgenössische Psychologie zwar überzeugt. Ob Ernst Machs Gestaltpsychologie, ${ }^{35}$ Sigmund Freuds Psychoanalyse $^{36}$ oder Karl Jaspers' Psychopathologie von 1913 - sie alle halten das Unbewusste in der menschlichen Seele gegenüber dem rationalen Anteil für dominant, meinen, es sei für das Gefühlsleben und die aus diesem resultierenden (affektiven) Handlungen bestimmend und als Unbewusstes nicht dechiffrierbar. ${ }^{37}$ Musil

32

David Wachter: Konstruktionen im Übergang. Krise und Utopie bei Musil, Kracauer und Benn, Freiburg im Breisgau, Berlin, Wien 2013, 83.

R. Musil: Quantificierbarkeit der Moral, 1308.

R. Musil: Das Unanständige, KA IX 136.

Ernst Mach: Analyse der Empfindungen und das Verhältnis des Physischen zum Psychischen, 1886; zum Verhältnis Musils zu Machs Gestaltpsychologie vgl. Karen Brüning: Die Rezeption der Gestaltpsychologie in Robert Musils Frühwerk, Frankfurt am Main 2015.

Sigmund Freud: Totem und Tabu, 1912, Das Ich und das Es, 1923; vgl. O. Krämer: Denken erzählen, 81-83.

Zu Mach und zur Gestalttheorie vgl. O. Krämer: Denken erzählen, 53-58 u. 61-65, sowie Florence Vatan: Gestalttheorie, in: B. Nübel, N. Ch. Wolf: 
jedoch nimmt einen im Vergleich mit Mach, Freud und Jaspers radikaleren Standpunkt ein: Er behauptet, es gebe keinen qualitativen Unterschied zwischen einem Sexualmörder und einem psychisch Gesunden hinsichtlich der Verknüpfung des Ichs mit dessen Handlungen.

(Psychisch) krank - entartet/ degeneriert - lebensunwürdig lebensunwert

Indem Musil behauptet, es gebe keinen qualitativen Unterschied zwischen einem Sexualmörder und einem psychisch Gesunden hinsichtlich der Verknüpfung des Ichs mit dessen Handlungen, steht er absolut ausserhalb des damaligen Mainstreams. Denn er wendet sich mit seiner Behauptung gegen die um 1910 bereits mächtige Psychoanalyse Freuds; ${ }^{38}$ noch dezidierter jedoch bezieht er Position gegen die zur Zeit seines Essays bereits weit verbreitete Annahme, dass ein enger Konnex zwischen Psychopathologie einerseits, Entartung und moderner Kunst andererseits bestehe - so das ziemlich einhellige Ergebnis einer ab dem Ende des 19. Jahrhunderts nicht nur in der deutschen Psychiatrie intensiv geführten Diskussion und zugleich der Marginalisierung und Pathologisierung

Robert Musil Handbuch, 531-537, hier 534: Bereits Mach habe die «Unrettbarkeit des Ich» verkündet. «In seiner Kritik am Ideal eines souveränen, vernunftgeleiteten Subjekts betont Musil die Auflösungstendenzen innerhalb des Ichs. Doch lehnt er Machs Definition des Ichs als eines fiktiven und prekären Bündels von Empfindungen ab. Demgegenüber beschreibt Musil das Ich als eine dynamische Gestalt, die ein breites Spektrum von möglichen Zuständen erleben kann: vom Zustand grosser Prägnanz bis zum labilen Zustand des ekstatischen Selbst. Darüber hinaus stellt er die traditionelle Vorstellung des Ich als zentraler und regulativer Instanz in Frage.» Zur Rolle Machs in Musils Denken und Musils kritischer Auseinandersetzung mit Mach vgl. Hans-Joachim Pieper: Musils Philosophie. Essayismus und Dichtung im Spannungsfeld der Theorien Nietzsches und Machs, Würzburg 2002, bes. 10-16 u. 128139. Y. Wübben: Psychiatrie, 525 . 
nicht in die Norm des Mainstreams passender künstlerischer Ausdrucksweisen. ${ }^{39}$

Unter Entartung verstehen Bénédict Augustin Morel, ${ }^{40}$ Cesare Lombroso $^{41}$ und Max Nordau ${ }^{42}$ krankhafte Abweichungen von der Norm, die nicht nur physische, sondern ebenso geistige und moralische Ursachen hätten; derartige Entartungen oder Degenerationen beträfen sowohl einzelne Personen wie auch deren Familien, ja, ganze Rassen und innerhalb der Gesellschaft besonders Künstler und Intellektuelle. ${ }^{43}$

Der weitestgehend einhelligen moralischen Verurteilung moderner Kunst als degeneriert und entartet um 1900 in der Nachfolge

Vgl. zum medizin- und rechtshistorischen Diskurs um 1900 Andrea Adams: Psychopathologie und «Rasse». Verhandlungen «rassischer» Differenz in der Erforschung psychischer Leiden (1890-1933), Bielefeld 2013; HeinzPeter Schmiedebach (Hg.): Entgrenzungen des Wahnsinns. Psychopathologie und Psychopathologisierungen in Urbanen und Provinziellen Öffentlichen Räumen um 1900, Berlin 2016; M. Ralser: Das Subjekt der Normalität, bes. 59-132 («Aus disparaten Figuren Fälle von Krankheit»); zur gerichtlichen Psychopathologie um 1900 vgl. das Standardwerk von Richard von Krafft-Ebing: Lehrbuch der gerichtlichen Psychopathologie. Mit Berücksichtigung der Gesetzgebung von Österreich, Deutschland und Frankreich, 3., umgearbeitete Auflage, Ausg. 2, mit einem Nachtrag: Die zweifelhaften Geisteszustände vor dem Civilrichter des deutschen Reiches nach Einführung des bürgerlichen Gesetzbuchs, Stuttgart 1900. Zur Biologisierung und pathologischen Psychologisierung des Künstlers vgl. Bettina Gockel: Die Pathologisierung des Künstlers. Künstlerlegenden der Moderne, Berlin 2010, 25-74.

Bénédict Augustin Morel: Traité des dégénérescences physiques, intellectuelles et morales de l'espèce humaine, Paris 1857.

Vgl. Cesare Lombroso: Entartung und Genie. Neue Studien, mit 12 Tafeln, gesammelt und unter Mitwirkung des Verfassers deutsch herausgegeben von Dr. Hans Kurella und G. H. Wigand, Leipzig 1894, in: Zeitschrift für Psychologie und Physiologie der Sinnesorgane 9 (1896).

42 Max Nordau: Entartung, hg. von Karin Tebben, Berlin 2013 (1. Aufl., I-II, 1892-1893).

43 Vgl. Steffen Krämer: Entartung in der Kunst. Die Verbindung von Psychopathologie und moderner Kunst von der Mitte des 19. Jahrhunderts bis zum Nationalsozialismus, 1-22, Kunstgeschichte. Open Peer Reviewed Journal: https://www.kunstgeschichte-ejournal.net/383/1/Krämer Entartung in der Kunst.pdf 
von Morel, Lombroso und Nordau stehen einige wenige Psychiater entgegen, die Werke psychisch Kranker als Kunst bewerten ${ }^{44}$ - seit 1945 durch Jean Dubuffet als Art brut bekannt ${ }^{45}$ - und die psychisch Kranken als Künstler ${ }^{46}$ wahrgenommen wissen wollen, ${ }^{47}$ wie zum Beispiel Hans Prinzhorn mit seiner Sammlung Bildnerei der Geisteskranken von $1922^{48}$ oder Walter Morgenthaler mit seinem 1921 erschienenen Buch Ein Geisteskranker als Künstler über den 1895 mit der Diagnose Schizophrenie in die Irrenanstalt Waldau bei Bern eingelieferten Adolf Wölfli. Aber diese Perspektive bleibt ohne Folgen für die Psychiatrie. ${ }^{49}$

$44 \quad$ Vgl. Alfred Bader (Hg.): Geisteskrankheit, bildender Ausdruck und Kunst. Eine Sammlung von Texten zur Psychopathologie des Schöpferischen, Bern, Stuttgart 1975. Die Problematik begrifflicher Unterscheidung zwischen der Kunst von psychisch Kranken und psychisch Gesunden präzise auf den Begriff bringt Ernst Klee: Wie «verrückt» ist Kunst? Prinzhorn: Die grösste Sammlung «pathologischer» Bilder: Hochzeit der Farben, in: Zeit, 10. Oktober 1980: «Den Streit darüber zu führen, was Kunst der «Geisteskranken〉 von der Kunst der «Normalen` unterscheide, ist selber schon eine verrückte Diskussion. Denn wer ist normal? Der Künstler mag - nach gesellschaftlichen Massstäben - ver-rückt sein; amtlich verrückt wird er erst, wenn ihn die Psychiatrie, als Hüterin einer konservativen Ordnung, mit einem psychiatrischen Etikett versehen hat. Dann können seine Arbeiten noch so gelungen sein, dann unterliegt er immer der Gewalt der Diagnose. Er bleibt ein Geisteskranker, mag er als Künstler noch so Hervorragendes leisten.»

https:// www.zeit.de/1980/42/hochzeit-der-farben/komplettansicht Vgl. Leo Navratil: Bilder im Kopf. Die Kunst aus der Abgeschiedenheit der Anstalt: https:/ / folio.nzz.ch/1996/september/bilder-im-kopf

48 Die Sammlung Prinzhorn, Einrichtung des Universitätsklinikums Heidelberg: https:// prinzhorn.ukl-hd.de/sammlung-prinzhorn/ Zu Hans Prinzhorns Kriterien der Auswahl für seine Sammlung vgl. Jörg Katerndahl: «Bildnerei von Schizophrenen». Zur Problematik der Beziehungssetzung von Psyche und Kunst im ersten Drittel des 20. Jahrhunderts, Hildesheim, Zürich, New York 2005, 45-89.

49 Vgl. zur Patientenkunst in der Irrenanstalt Rheinau: https:// www.nzz.ch/zuerich/kopie-von-zuercher-patientenkunstbleibt-im-keller-ld.1375737 
Bereits denken 1920 deutsche Juristen und Psychiater über die Euthanasie $^{50}$ angeblich «leere[r] Menschenhülsen ${ }^{51}$ nach, aus ihrer Sicht eine straffreie «Erlösungstat $»^{52}$ - gemeint sind genau jene Langzeitpatienten, für deren schöpferische Intelligenz Prinzhorn mit Sammlung und Buch ein Forum eröffnet hatte. ${ }^{53}$ So sprechen Karl Binding, Professor für Öffentliches Recht in Leipzig, und Alfred Hoche, seit 1902 Ordinarius für Psychiatrie in Freiburg, in ihrem Buch Die Freigabe der Vernichtung lebensunwerten Lebens behinderten und psychisch kranken Menschen ihr Menschsein ab..$^{54}$ Nicht erst im

https:/ / www.nzz.ch/zuerich/klinik-rheinau-irrenkunst-ans-licht-geholtld.1293534 Zur Patientenkunst in der ehemaligen Landes- und Pflegeanstalt Niedernhart: Hemma Schmutz, Brigitte Reutner (Hg.): Patientinnenkunst aus der ehemaligen Landesheil- und Pflegeanstalt Niedernhart 1875-1925, Weitra 2019.

50 Vgl. Michael Burleigh: Death and Deliverance. «Euthanasia» in Germany c. 1900-1945, Cambridge 1995.

51 Karl Binding, Alfred Hoche: Die Freigabe der Vernichtung lebensunwerten Lebens. Ihr Mass und ihre Form, Leipzig 1920, 55.

52 Ebd., 28.

53 Vgl. Udo Benzenhöfer: Der gute Tod? Geschichte der Euthanasie und Sterbehilfe, Göttingen 2009; Michael Wunder: «Auf dieser schiefen Ebene gibt es kein Halten mehr», in: Michael Wunder, Ingrid Genkel, Harald Jenner (Hg.): Auf dieser schiefen Ebene gibt es kein Halten mehr. Die Alsterdorfer Anstalten im Nationalsozialismus, Stuttgart ${ }^{32} 2016$ (1. Aufl. 1987) 35-78.

54 Lars Winkler: «Die Freigabe der Vernichtung lebensunwerten Lebens». Eine unheilvolle Schrift und ihr Jahrhundert, in: Freischüssler 16 (2008) 63-64, hier 63: «Als wissenschaftlich formulierte Grundlage einer auf radikaler Kosten-Nutzen-Rechnung getrimmten Volkswirtschaft und Verteidigungsschrift der daraus resultierenden Beurteilung des Wertes und 〈Unwertes〉 von Menschen ist die geistige Urheberschaft der Schrift Hoches und Bindings an den Verbrechen der Nazis ab 1933 nicht zu verkennen. Die Schrift bereitete die Verbrechen der Nazis in erheblichem Masse vor. Nicht nur terminologische Gemeinsamkeiten sind offensichtlich. Karl Binding und Alfred Erich Hoche haben ihre wissenschaftliche Verantwortlichkeit in hohem Masse missbraucht und gezeigt, dass Wissenschaft in einem radikal-ökonomisierten Umfeld zum Instrument jeglicher, anfangs vermeintlich ökonomisch begründeter Politik führen kann.» http:/ / akj.rewi.hu-berlin.de/zeitung/08-16/material/fs16-16-unheilvolleschrift.pdf 
110 Ulrike Zeuch: Das Gesunde und Kranke in der Literatur

Dritten Reich wird der Kunst der Moderne der Kampf, kulminierend in der Wanderausstellung «Entartete Kunst» (1937-1941), angesagt. ${ }^{55}$

\section{Musils Axiom: Transformation des «Kranken» und «Unanständigen» durch Kunst}

Musil macht, wie wir gesehen haben, geltend, dass durch die Darstellung des Kranken und Unanständigen in der Kunst dieses transformiert werde und als in die Kunst Transformiertes nicht mehr unanständig oder krank sei. Die qualitative Transformation des Unanständigen und Kranken in etwas, das diese Merkmale nicht mehr aufweist, muss Musils Argumentation zufolge mit der Darstellung oder genauer: mit der Art der Darstellung zu tun haben. Was also meint Musil mit künstlerischer Darstellung?

Es heisst etwas darstellen: seine Beziehungen zu hundert andern Dingen darstellen; weil es objektiv nicht anders möglich ist, weil man nur so etwas begreifbar und fühlbar machen kann, ... wie ja auch wissenschaftliches Verständnis nur durch Vergleichen und Verknüpfen entsteht, wie menschliches Verstehen überhaupt entsteht. Und wenn auch diese hundert anderen Dinge wieder unanständig und krank wären: die Beziehungen sind es nicht, das Auffinden von Beziehungen ist es niemals. [...] die Kunst sucht Wissen; sie stellt das Unanständige und Kranke durch seine Beziehungen zum Anständigen und Gesunden dar, das heisst nichts anderes als: sie erweitert ihr Wissen vom Anständigen und Gesunden. ${ }^{56}$

Etwas künstlerisch darzustellen, heisst für Musil demzufolge, der dem Menschen innewohnenden curiositas zu folgen, die, wenn sie sich über Grenzen des Erlaubten, über Tabus hinwegsetzt, dasjenige, was bis dato isoliert für sich gestanden hat, als etwas mit vielem, ja im Grunde genommen mit allem Korrelierendes, Verbundenes konstruiert und als solches zum Ausdruck bringt, visualisiert

55 Vgl. Jan Eric Schulte: Mit Gas gegen «leere Menschenhülsen». Kranke und Behinderte waren die Opfer des ersten nationalsozialistischen Massenmords. https: / / www.zeit.de/zeit-geschichte/2017/01/euthanasie-ns-regimekranke-behinderte-massenmord 
und verbalisiert. ${ }^{57}$ Dieses Konstruieren von Beziehungen mündet, so Musil, in Wissen. Wissen meint hier: Vielansichtigkeit, Vernetzung, Multiperspektivität, Absage an eine verbindliche Wahrheit, an die gültige Lesart, Bekenntnis zur Relativität des Standpunktes. Wahr werde dieser Standpunkt schon allein dadurch, dass der Betrachter aufgebe, auf der Richtigkeit seines Blicks zu bestehen. Wahr werde dieser Standpunkt zudem dadurch, dass er sämtliche nur möglichen anderen Standpunkte integriere und als ebenso gültige zulasse.

Dass das Zulassen dieser anderen Standpunkte möglicherweise beliebig sein und sogar dazu führen könnte, auch kontradiktorische Standpunkte zu integrieren - diese Möglichkeit zieht Musil an dieser Stelle nicht in Betracht. Begriffe wie «fühlbar» und «begreifbar» können nur dann, wie Musil dies tut, als miteinander kompatibel angesehen werden, wenn das Begreifen des Begreifbaren als sensueller, nicht als rationaler Vorgang verstanden wird.

Musil setzt in seinem Konzept der künstlerischen Darstellung Wahrheit mit Ganzheitlichkeit, Richtigkeit mit Totalität oder AllAnsichtigkeit aller nur denkbaren Perspektiven und Objektivität mit Fühlbarkeit gleich. Indem er dies tut, ist er derselben erkenntnistheoretischen Problematik erlegen wie vor ihm bereits um 1800 Herder und die Romantiker, etwa Friedrich Schlegel: im Moment der ersehnten Ganzheitlichkeit, Totalität oder All-Ansichtigkeit, im Augenblick reinen Fühlens über kein Kriterium der Beurteilung mehr zu verfügen, das es erlauben würde, das Gefühlte zu bewerten; die Preisgabe rationaler Beurteilungskriterien ist ja gerade die Voraussetzung beziehungsweise der Preis dafür, überhaupt in einen solchen Zustand allumfassender Bezugshaftigkeit gelangen zu können. In nuce ist Musils Konzept der künstlerischen Darstellung identisch mit dem romantischen Postulat der unendlichen Vermischung, mit Herders sensorium commune. Alles ist eins:

57 D. Wachter: Konstruktionen im Übergang, 87: «Mit seinem erklärten, häufig provokativ gezeichneten Überbietungsethos macht sich der Protagonist (sc. Ulrich in der MoE) die Gegenwartsgesellschaft als Ordnungsvakuum, als tabula rasa und Konstruktionsfeld gedanklich zunutze.» 
Wahrnehmung, Gefühle, Intentionen, Gedanken. ${ }^{58}$ Das Ergebnis ist eine meist nur ungenaue Vorstellung des zu schildernden Vorganges, aber ringsherum ein dunkles Klingen seelischer Verwandtschaften, ein langsames Bewegen weiter Gefühls-, Willens- und Gedankenzusammenhänge. ${ }^{59}$

Dieses dunkle Klingen seelischer Verwandtschaft erfolgt im Zweifelsfall sogar, wie im ersten Teil von $M o E$, zwischen Ulrich und dem Sexualmörder Moosbrugger. Vom Erzähler so konstruiert, fungiert «die Figur, die von allen genommen ist, als Projektion aller»; Moosbrugger wird zur Kipp- oder Inversionsfigur. ${ }^{60}$ Die Bewertung kippt, je nach Ansicht und eingenommener Perspektive: Moosbrugger ist ein Sexualmörder und / oder der wahre, der neue Mensch jenseits von Gut und Böse, ${ }^{61}$ in diesem Punkt treffen sich

58 Vgl. hierzu U. Zeuch: Umkehr der Sinneshierarchie, 142-162; dies.: Das Unendliche - Höchste Fülle oder Nichts? Zur Problematik von Friedrich Schlegels Geist-Begriff und dessen geistesgeschichtlichen Voraussetzungen, Würzburg 1991, 97-120.

59 R. Musil: Das Unanständige, KA IX, 140; dieses dunkle Klingen, Allgefühl, Mit-allem-Fühlen, diese Verwandtschaft der menschlichen Seele mit allem, was ist, findet sich präfiguriert bei Rainer Maria Rilke in seinem Gedichtband Mir zur Feier von 1897 / 98, in: Gedichte 1895-1910, hg. von Manfred Engel, Ulrich Fülleborn, Frankfurt am Main 1996, 66-111, verdichtet in der Aussage des lyrischen Ichs: Alles ist Eins.

60 Vgl. Walter Fanta: Krieg. Wahn. Sex. Liebe. Das Finale des Romans «Der Mann ohne Eigenschaften» von Robert Musil, Klagenfurt 2015. Fanta hat in seinen Studien zur Textgenese in Bezug auf die beiden Figuren Ulrich und Moosbrugger gezeigt, dass sich die Konstellation im weiteren Verlauf im «apokryphen Moosbrugger II» (200) transformiert: Moosbrugger wird zur «wilden, eingesperrten Möglichkeit einer gefährlichen Handlung»; «Der Erzähler (Gott) lässt Moosbrugger tanzen. Tanz, Aufbewahrung und Auflösung Moosbruggers vertreten trotzdem zugleich etwas im Seinsfeld Ulrichs, das dort tanzt, aufbewahrt und aufgelöst wird, in Verbindung mit dem Baum der Gewalt.» «Moosbrugger ist Ulrichs Phantom.» (ebd.)

61 Zur Bedeutung von Nietzsches Perspektivismus als Ausdruck generellen Zweifels «an der Möglichkeit unbedingt wahrer Erkenntnis» für Musil vgl. H.-J. Pieper: Musils Philosophie, 46-60, hier 47. Zur Bedeutung des Experiments bei Nietzsche und Musil, vgl. Andrea Pelmter: «Experimentierfeld des Seinkönnens» - Dichtung als «Versuchsstätte». Zur Rolle des 
Moosbrugger und Ulrich; der wahre Moosbrugger ist noch nicht in die Gesellschaft eingetreten, deren Moral falsch, verlogen, scheinheilig, brüchig, krank ist, und Ulrich verlässt als eben der Mann ohne Eigenschaften ganz bewusst diese falsche und kranke Gesellschaft und macht sich zum Aussenseiter, der nach mystischer Erfahrung sucht, eine Utopie, eine «Wirklichkeit bzw. Wahrheit hinter den Wahrnehmungskonventionen der Welt». ${ }^{62}$ Insofern ist es für die Funktion Moosbruggers als Kipp- und Inversionsfigur irrelevant, ${ }^{63}$ ob Musil Moosbrugger «mit den Symptomen der Dementia praecox und Schizophrenie ausgestattet» hat oder nicht - eine Frage, die die Forschung nicht nur lange beschäftigt, sondern heute nicht befriedigend beantwortet hat. ${ }^{64}$

Experiments bei Robert Musil, Würzburg 2008, 81-93; Pelmter hebt vor allem Nietzsches Konzept der «Selbstexperimentation, der Anwendung der experimentellen Methode auf die eigene Person» (92) in seiner Bedeutung für Musil hervor. Kunst (Lebenskunst und/oder ästhetische Produktion) versteht Musil mit Nietzsche als Ort, «in welchem die Experimentalphilosophie, das Experimentieren mit sich selbst und die Schaffung von neuen Lebensentwürfen, durchgeführt werden kann» (93). Martina Wagner-Egelhaaf: Mystik, in: B. Nübel, N. Ch. Wolf: Robert Musil Handbuch, 705-710, hier 705.

63 Vgl. W. Fanta: Krieg. Wahn. Sex. Liebe: Zwar werde Moosbrugger im ersten Teil von $M o E$ «vom Erzähler als Grenzfall-Objekt des juridisch-ethischen Diskurses in den Roman ein- und später in der Schilderung seiner Biographie in den soziologischen bzw. auch psychopathologischen Diskurs überführt» (199). Aber da die Figur als archaisches Vorbewusstsein, das der zeitgenössischen Kultur fehle, konzipiert sei, sei die Frage nach den Quellen, die Musil verwendet, «bei solcher Sicht Moosbruggers im Grunde irrelevant [...]» (199).

64 Y. Wübben: Psychiatrie, 529: «Trotz zahlreicher Arbeiten, die Musils literarische Texte psychiatriehistorisch einordnen, bleibt hier noch vieles zu tun: Unbeantwortet ist etwa die Frage, welche psychiatrischen Diskussionen und Problemlagen (Klassifikation, Erbbiologie, Sprachforschung) Musil im Einzelnen rezipiert hat.» 
Welche Konsequenzen hat Musils Axioms für die Kunst?

Jede Perversität lässt sich darstellen. Sie lässt sich darstellen durch ihren Aufbau aus Normalem, da man die Darstellung sonst nicht verstände. ${ }^{65}$ Zunächst scheint es so, als meine Musil, dass der Künstler oder Schriftsteller das Perverse, Abweichende, Kranke gezielt aus dem Normalem konstruiere. Dies suggeriert die Formulierung «lässt sich darstellen», so als sei es ins Belieben des Künstlers gestellt, wie er Abweichungen darstelle. Demnach wäre das Kranke an sich nicht zwingend aus Normalem aufgebaut. In Wahrheit gibt es keine Perversität beziehungsweise Unmoral, der nicht auf irgendeine Weise Gesundheit beziehungsweise Moral korrelierte. Das setzt voraus, dass sich zu allen Bestandteilen, aus denen sie sich aufbaut, analoge Elemente auch in der gesunden und zusammenlebenstüchtigen Seele finden. ${ }^{66}$

Aber Musil meint noch etwas Anderes: In jeder sich als gesund und damit als normal betrachtenden Seele, in jedem sich als normal betrachtenden Menschen steckt etwas, das mit dem NichtNormalen korreliert, das heisst sich in einer Wechselbeziehung mit ihm befindet. Sonst würde man es weder darstellen noch verstehen können. Es gibt also einen Nucleus, etwas Kranken wie Gesunden Gemeinsames, dem es sich anzunähern gilt, will man wirklich begreifen, was und wer der Mensch ist.

Nicht nur sprengt diese von Musil formulierte Einsicht den äusserst sorgsam aufrechterhaltenen und gesicherten Bereich der Normalität; sie ist - als Aufforderung an die Kunst und Literatur gefasst, solches Gemeinsames darzustellen - zugleich radikal, und zwar sowohl hinsichtlich des «Gegenstandes» wie der Darstellbarkeit. ${ }^{67}$ Wie radikal Musils Einsicht ist, zeigt sich im Kontext des Unsagbarkeitstopos nach dem Holocaust: Die Grenze ist markiert

65 R. Musil: Das Unanständige, KA IX 143.

66 Ebd., 142-143.

67 Bis heute werden Sexualstraftäter gemeinhin als Monster bezeichnet, so etwa Josef Fritzl als «Monster von Amstetten», in: Die Zeit, Nr. 48, 21. 
durch die Denkunmöglichkeit (oder das Denkverbot), in dem Täter, dem Verursacher millionenfacher Ermordung von Menschen, dennoch den Menschen, einen Menschen, auch einen Menschen sehen zu sollen oder gar zu müssen und ihn als einen solchen darzustellen. ${ }^{68}$

Die Radikalität ist jedoch auch in eine andere Richtung denkbar: Statt über die Abweichung von den gesellschaftlichen Moralvorstellungen in die Handlungsverweigerung oder Handlungsagonie und in die Utopie des Tausendjährigen Reiches ${ }^{69} \mathrm{zu}_{\text {flüchten, }}{ }^{70}$ wie Ulrich und Agathe dies im anderen Zustand tun, ${ }^{71}$ ist diese Radikalität als Aufforderung zu verstehen, aktiv das zu zerstören, was einen zerstört. In seiner Musik in Bildern nach dem Märchen von Hans Christian Andersen, Das Mädchen mit den Schwefelhölzern, ergänzt Helmut Lachenmann dieses Märchen u.a. durch einen Brief der 1977 im Hochsicherheitsgefängnis Stammheim durch

November 2019, Dossier, 18: «Es gibt eine Grenze des Erträglichen. Fast zwei Jahrzehnte lang hat die Psychiaterin Heidi Kastner Mörder und Vergewaltiger begutachtet, auch Josef Fritzl, das Monster von Amstetten. So langsam reicht es ihr.»

68 Ulrike Zeuch: Krise der Repräsentation oder: Welche Wirklichkeit soll Literatur darstellen, in: Komparatistik 6 (2005) 113-129.

69 Vgl. Robert Leucht: Utopie, in: B. Nübel, N. Ch. Wolf: Robert Musil Handbuch, 725-730, zur Vieldeutigkeit von Musils Utopiebegriff und zur bislang nicht geklärten Frage seiner «wissens- und diskursgeschichtlichen Kontextualisierung» (727). D. Wachter: Konstruktionen im Übergang, 129, deutet die Ekstase des anderen Zustands, dessen taghelle Mystik, im Kapitel «Ins Tausendjährige Reich» (MoE 670) als Musils Versuch, die Krise der Moderne, die sich vor allem in ihrer, so Musil, fehlgeleiteten «Reduktion von metaphorischer Mehrdeutigkeit auf begriffliche Ordnung» zeige, zu bewältigen, ja zu überwinden.

70 D. Wachter: Konstruktionen im Übergang, 158 bemerkt zu Musils Utopie des Tausendjährigen Reiches m.E. zu Recht kritisch an: «Mit seinen Enthaltungsgesten bringt er eine strategische Indifferenz hervor, die Denkoptionen durchspielt und verdichtet, ohne sie als Programm auf Dauer zu stellen».

71 Martina Wagner-Egelhaaf: Anderer Zustand, in: B. Nübel, N. Ch. Wolf: Robert Musil Handbuch, 710-712, hier 711: «Der andere Zustand legitimiert von den gesellschaftlichen Moralvorstellungen abweichende Handlungsweisen [...].» 
Selbstmord gestorbenen Gudrun Ensslin ${ }^{72}$ und wirft dabei die Frage auf: Wäre aus dem Mädchen des Märchens, von einer eiskalten und gleichgültigen, mit sich selbst beschäftigten Gesellschaft im Stich gelassen, wenn es die Silvesternacht überlebt hätte, später einmal eine Terroristin geworden?

Gudrun Ensslin (1940-1977)

der kriminelle, der wahnsinnige, der selbstmörder - sie verkörpern diesen widerspruch. sie verrecken in ihm. ihr verrecken verdeutlicht die auswegslosigkeit/ohnmacht des menschen im system: entweder du vernichtest dich selbst oder du vernichtest andere, entweder tot oder egoist. in ihrem verrecken zeigt sich nicht nur die vollendung des systems: sie sind nicht kriminell genug, sie sind nicht wahnsinnig genug, sind nicht mörderisch genug, und das bedeutet, ihren schnelleren tod durch das system im system. in ihrem verrecken zeigt sich gleichzeitig die verneinung des systems: ihre kriminalität, ihr wahnsinn, ihr tod ist ausdruck der rebellion der zertrümmerten subjekte gegen ihre zertrümmerung, nicht ding, sondern mensch. (schreibt auf unsere haut.)

Anfang 1975

Aber vielleicht sind diese beiden Denkmöglichkeiten, Handlungsagonie und radikaler Aktionismus, im Kern gar nicht so verschieden? Jedenfalls weist die jüngere Musil-Forschung in diese Richtung, wenn sie sich fragt, inwiefern «auch die essayistische Utopie des Romans eine Keimzelle des Terrors» enthalte. ${ }^{73}$ Der Krieg, der zeigt, dass «U[1rich]s System [...] am Ende desavouiert» ist, wird zur Option einer «Flucht aus dem Frieden ${ }^{74}$ und «bildet ein Zerrbild des anderen Zustandes, das zugleich aus dessen dystopischen Potenzialen resultiert, die wiederum mit

72 https://www.tip-berlin.de/interview-mit-dem-komponisten-helmutlachenmann/

Zuletzt vgl. Opernhaus Zürich:

https: / / www.opernhaus.ch/spielplan/kalendarium/das-maedchenmit-den-schwefelhoelzern/2019-2020/

73 D. Wachter: Konstruktionen im Übergang, 160; zum historischen Kontext der Zwischenkriegszeit vgl. Hans Feger, Hans-Georg Pott, Norbert Christian Wolf (Hg.): Terror und Erlösung. Robert Musil und der Gewaltdiskurs in der Zwischenkriegszeit, München 2009.

74 Vgl. R. Musil: MoE, hg. von Adolf Frisé (aus dem Nachlass), II 1844. 
utopischen Energien bis zur Ununterscheidbarkeit vermengt sind». ${ }^{75}$ «Vom Krieg», so Fanta, «steht fest, dass Ulrich freiwillig in ihn eintreten wird.» ${ }^{76}$

In seinem Essay diagnostiziert Musil die Krise der Moderne als eine Krise rationaler Beurteilung, Bewertung, Erklärbarkeit. Das war damals nicht neu. Musil appliziert die Krise rationaler Beurteilung auf die Unterscheidung zwischen Krankem und Gesundem. Auch dies ist nicht neu. Was aus seinen Reflexionen über das Ungesunde und Kranke in der Kunst resultiert, ist eine Haltung, dass nichts Menschliches dem Menschen fremd sei. Auch dieser Gedanke, der sich bereits bei Terenz findet, ist nicht neu: «homo sum, humani ni[hi]1 a me alienum puto». ${ }^{77}$ Aber im Kontext der Zeit, vor und nach dem Ersten Weltkrieg, im Kontext einer den Menschen verachtenden Ideologie des «Gesunden» als Kriterium für Selektion und deren konsequenten Umsetzung in Euthanasie und Genozid ist es ein Statement, das von der geistigen, intellektuellen Solidarisierung mit allem, was dem Denken zunächst fremd scheint, zeugt.

Folgt man Musils Argumentation, führt diese jedoch - und das ist der problematische Aspekt seines Essays - dazu, dass die begrifflichen Konturen verschwimmen, sich auflösen «wie modrige Pilze», so Lord Chandos im Brief von Hugo von Hofmannsthal, ${ }^{78}$ dass die Orientierung abhanden kommt, die Entschlossenheit, etwas Bestimmtes zu tun und anderes zu lassen - alles Schlüsselbegriffe und Stichworte für die Beschreibung des anderen Zustandes im MoE -,

75 D. Wachter: Konstruktionen im Übergang, 161.

76 W. Fanta: Krieg. Wahn. Sex. Liebe, 177; vgl. das ganze Kapitel «Mobilisierung im Hinterkopf», 174-183. Zu Ulrich, der «seinen Mann steht», seine Männlichkeit beweist, indem er in den Krieg zieht, vgl. Ulrich Boss: Männlichkeit als Eigenschaft. Geschlechterkonstellationen in Robert Musils Der Mann ohne Eigenschaften, Berlin 2013, 1-29.

77 Ter. Heaut. 77.

78 Vgl. Ulrike Zeuch: «[...] die abstrakten Worte [...] zerfielen mir im Munde wie modrige Pilze». Zum Verlust des Gegenstandes in der Literaturtheorie seit 1966, in: Euphorion 95/1 (2001) 101-121. 
da die Handlung stagniert, sich in nicht endende Reflexionen auflöst, sich im Kreis dreht und der Krieg dann doch als eine wenn auch schlechte Option einer Flucht aus dem Frieden winkt.

Ist also eine Unterscheidung zwischen «krank» und «gesund» ethisch unverzichtbar, um handlungsfähig zu sein und zu bleiben, um die Aporie zu vermeiden, in die Musils Essay gedanklich führt, um den von Musil im MoE beschriebenen und historisch stattgefundenen Machtmissbrauch zukünftig zu verhindern? Die begriffliche Unterscheidung ist, wie ich meine, unverzichtbar: Das Gesunde ist nicht das Kranke. Aber ebenso unverzichtbar ist - und darin zeigt sich meines Erachtens die Aktualität von Musils Essay -, sich immer und immer wieder neu $\mathrm{zu}$ fragen: Wer bestimmt aus welchem Grund zu welchem Zweck, wer gesund und wer krank ist? Was ist jeweils mit «gesund» und «krank» gemeint, in welchem Kontext medizinisch, psychiatrisch, religiös, moralisch - werden diese Begriffe gebraucht oder missbraucht? Für diese Fragen sensibilisiert zu sein und missbräuchliche Verwendung zwecks Ausgrenzung und Selektion in Literatur, Film, Musik, also in der Kunst kritisch darzustellen, den Missbrauch publik zu machen - das ist Musils Essay gedanklich geschuldet und zu verdanken. Allerdings gibt Musil systemimmanent konsequent - keine Antwort auf die Frage nach möglichen Kriterien, die eine Unterscheidung zwischen «gesund» und «krank» erlaubten. ${ }^{79}$

Zur Bestimmung seelischer Gesundheit heute vgl. Wolfgang Rissmann (Hg.): Was heisst seelische Gesundheit? «Körper» - «Seele» - «Geist», Würzburg 2011; interessanterweise wird in der Materialsammlung der WHO zur psychischen Gesundheit der Begriff «Psychische Gesundheit» vorausgesetzt, ohne zu erklären, was mit ihm gemeint ist; psychische Krankheiten (psychische Behinderungen, psychische Störungen, geistiges Unvermögen, geistige Umnachtung) hingegen werden differenziert und ausführlich dargelegt (20-27):

https://www.who.int/mental health/policy/who resource book german.pdf 
119 Ulrike Zeuch: Das Gesunde und Kranke in der Literatur

conexus $3(2020)$ 96-119

(C) 2020 Ulrike Zeuch. Dieser Beitrag darf im Rahmen der Lizenz CC BYNC-ND 4.0 - Creative Commons: Namensnennung/nicht kommerziell/ keine Bearbeitungen - weiterverbreitet werden.

\section{cc) (i) (2)}

https:// doi.org/10.24445/ conexus.2020.03.008

Prof. Dr. Ulrike Zeuch, Universität Zürich, Deutsches Seminar, Schönberggasse 9, 8001 Zürich

ulrike.zeuch@uzh.ch 